\section{Duração do aleitamento materno, regime alimentar e fatores associados segundo condições de vida em Salvador, Bahia, Brasil}

\author{
Breastfeeding duration, infant feeding \\ regimes, and factors related to living conditions \\ in the city of Salvador, Bahia, Brazil
}

Lucivalda P. Magalhães de Oliveira 1 Ana Marlúcia O. Assis 1 Gecynalda Soares da Silva Gomes 2 Matildes da S. Prado ${ }^{3}$ Maurício L. Barreto ${ }^{3}$

\footnotetext{
1 Escola de Nutrição, Universidade Federal da Bahia, Salvador, Brasil. 2 Instituto de Matemática, Universidade Federal da Bahia, Salvador, Brasil. 3 Instituto de Saúde Coletiva Universidade Federal da Bahia, Salvador, Brasil.

Correspondência L. P. M. Oliveira Escola de Nutrição, Universidade Federal da Bahia.

Rua Araújo Pinho 32, Salvador, $B A$ 40110-150, Brasil. lucipmo@ufba.br
}

\begin{abstract}
This cross-sectional study aimed to identify breastfeeding duration, infant feeding regimes, and factors related to living conditions among 811 children under 24 months of age in Salvador, Bahia, Brazil. Data were statistically analyzed by survival analysis, Pearson's chi-square test, and multivariate logistic regression. Median duration of exclusive, predominant, and total breastfeeding was 30.6, 73.0, and 131.5 days, respectively. Exclusive or predominant breastfeeding was discontinued in $83.6 \%$ of the subjects. Children with poor living conditions were 2.3 times more likely (95\%CI: 1.09-5.01) to receive early supplementary food, whereas the figure for the very poor increased to 2.5 (95\%CI: 1.20-5.34). Early exclusive or predominant breastfeeding discontinuation was associated with early pregnancy and poor living conditions of the children and their families. Programs directed towards proper breastfeeding and healthy feeding practices in childhood should consider the social factors associated with early introduction of supplementary foods in this population.
\end{abstract}

Child Welfare; Breast Feeding; Food Habits

\section{Introdução}

Na atualidade está sendo retomada a discussão em torno da iniqüidade em saúde e a busca de indicadores que melhor favoreçam a compreensão e resolução ou redução dos problemas de saúde nas populações pobres 1 . A busca da melhoria da saúde da população pobre tem motivado também a convergência de esforços de vários organismos internacionais 1,2 , com o renovado interesse pela diminuição das desigualdades em saúde 3 .

No escopo desse esforço, tem se destacado a saúde e nutrição na infância. A melhoria no acesso e na qualidade dos cuidados no pré-natal, no parto e no planejamento familiar, aliada à prática da alimentação saudável, em especial nos primeiros anos de vida, têm sido destacados como investimentos relevantes para a redução da carga de morbimortalidade na infância, especialmente entre as crianças de baixa renda 1 .

As evidências indicam que ações como promoção do aleitamento materno, utilização precoce e adequada do soro de reidratação oral, o cumprimento da agenda de vacinação, a vigilância nutricional e a melhoria do saneamento básico apresentam-se como intervenções eficientes na redução da taxa da morbimortalidade e garantia da sobrevivência da criança. Estima-se que intervenções com terapia de reidratação oral e aleitamento materno previnam mais 
de $10,0 \%$ das mortes de crianças menores de cinco anos de idade; quando são consideradas outras ações conjuntamente, este percentual pode chegar a $15,0 \% 4$.

Embora o aleitamento materno adotado exclusivamente nos seis primeiros meses de vida, e a partir daí complementado com alimentos disponíveis na unidade familiar até os dois anos de idade seja reconhecido como o padrão alimentar capaz de diminuir a morbimortalidade e de assegurar o crescimento físico adequado da criança 5, temos vários fatores ligados ao ambiente social e econômico familiar, aos serviços de saúde e às características biológicas inerentes à mãe e à sua criança 6 que interferem na adoção desse padrão alimentar e na duração do aleitamento ao peito.

Entende-se que o abandono da prática do aleitamento materno e a substituição total ou em parte do leite materno precocemente por outros alimentos é ainda mais nefasta quando adotada para as crianças pobres, uma vez que essas estão mais expostas a muitos agentes infecciosos, têm menor capacidade de resposta imunológica e menor chance de receberem as intervenções e cuidados que podem prevenir ou tratar até as doenças mais comuns 7.

Nesse sentido, a prática alimentar na infância, similarmente a outros eventos em saúde, guarda estreita relação com as condições de vida, com a definição e priorização das políticas públicas e com o poder e capacidade de escolha materna.

Assim, o presente estudo tem como objetivo conhecer a freqüência e a duração do aleitamento materno, os fatores associados à interrupção precoce do aleitamento exclusivo ou predominante, e a adoção dos diferentes regimes alimentares na perspectiva diferenciada das condições de vida das crianças menores de 24 meses de idade e de suas famílias na cidade de Salvador, Bahia, Brasil.

\section{População e método}

Este é um estudo transversal envolvendo 811 crianças menores de 24 meses de idade, que integraram a amostra de uma investigação mais ampla planejada para avaliar as condições de saúde e nutrição de pré-escolares na cidade de Salvador, intitulada Estudo Multicêntrico de Consumo Alimentar em Metrópoles Brasileiras, financiada pelo Ministério da Saúde 8.

O desenho amostral adotado no projeto inicial, acima referido, foi baseado em conglomerados em três estágios: o setor censitário é a unidade primária de seleção, o domicílio é a unidade secundária e a criança é a unidade terciária. Em caso de mais de uma criança menor de cinco anos no mesmo domicílio, era sorteada uma delas para compor a amostra.

A cidade de Salvador foi dividida em quatro quartis, conforme foi mapeada de acordo com o índice de condições de vida (ICV), segundo modelo proposto por Paim et al. (Paim JS, Silva LMV, Costa MCN, Prata PR, César ALM. Análise da situação de saúde no Município de Salvador segundo as condições de vida. Relatório final apresentado à Organização Pan-Americana da Saúde/Conselho Nacional de Desenvolvimento Científico e Tecnológico; 1995). Esse índice foi produzido com base em variáveis sócio-econômicas, escolaridade materna e do chefe da família, taxa de mortalidade infantil e acesso ao saneamento básico, distribuídos nos diferentes setores censitários da cidade de Salvador. Essas condições receberam pontuação crescente à medida que se tornavam mais adequadas. De acordo com essa pontuação a cidade foi dividida em quatro quartis de condições de vida (elevado, intermediário, baixo e muito baixo).

Assim, para cada quartil foram selecionados, ao acaso, 30 setores censitários, totalizando 120 setores, e em cada um deles foram sorteados 60 domicílios, visando a garantir um conjunto mínimo de aproximadamente 1.700 crianças a serem pesquisadas; número estimado com base nas prevalências dos déficits dos indicadores antropométricos altura/idade e peso/idade, respectivamente de $14,0 \%$ e $8,0 \%$, e com base na prevalência da anemia de $20,0 \%$.

No total, integraram a amostra final do estudo 1.792 crianças menores de cinco anos de idade, das quais 811 tinham de 0 a 24 meses de idade e compõem a amostra deste estudo. Todas as crianças menores de cinco anos de idade eram elegíveis para integrar a amostra do estudo, cuja participação esteve na dependência da concordância dos pais ou responsáveis pela criança.

Considerando que o desenho original do presente estudo não foi elaborado com a finalidade de investigar o padrão alimentar das crianças menores de dois anos de idade, calculou-se o poder da amostra (1- $\beta$ ), com o nível de significância $(\alpha)$ de $5 \%$, para detectar as principais associações de interesse. Assim, essa amostra apresenta poder de $78,0 \%$ para avaliar a associação entre a interrupção precoce do aleitamento exclusivo ou predominante e as condições sócio-econômicas, e de 89,0\% para identificar a relação entre as diferentes classes sociais e o regime alimentar adotado. A associação existente entre a orientação para o uso do 
primeiro leite oferecido à criança e o índice de condições de vida, é calculada com um nível de acerto de $99,0 \%$.

Os dados foram coletados por estudantes da graduação em nutrição, devidamente treinados sobre as técnicas de coleta, e supervisionados pelos investigadores principais. Foi realizado estudo piloto em um bairro da cidade, quando foram aplicados aproximadamente duzentos blocos de questionários, o que favoreceu o ajuste do instrumento e a definição da logística do trabalho de campo. Esse bairro foi excluído da amostra final do estudo. Nessa ocasião avaliouse também o desempenho dos estudantes e realizou-se a seleção dos entrevistadores.

\section{Aspectos éticos}

A participação da criança no estudo foi dependente da concordância dos responsáveis. Atendendo aos objetivos mais amplos desta investigação, foram realizados os diagnósticos do estado antropométrico e da anemia nas crianças que compõem a amostra do estudo. Assim, atendendo aos pressupostos éticos, em caso de desnutrição e de anemia, os resultados foram imediatamente repassados ao responsável e a criança foi referenciada ao serviço de saúde para o atendimento pediátrico e encaminhada também ao Serviço de Nutrição da Unidade de Saúde, integrando-a ao Programa de Atenção ao Desnutrido, vigente à época do estudo. Quando necessário, foi fornecida também ao responsável orientação sobre a alimentação da criança.

\section{Coleta de dados}

As mães ou responsáveis pela criança responderam, em seus domicílios, a um questionário previamente padronizado abrangendo questões sobre as características biológicas e sociais maternas, história gestacional e pós-parto, consumo alimentar da criança nas 24 horas que antecederam a entrevista, aleitamento materno e época de iniciação do consumo de alimentos diferentes do leite materno pela criança. A coleta de dados ocorreu em janeiro e fevereiro de 1996.

Os investigadores principais realizaram uma revisita a $10,0 \%$ do total dos domicílios, com o objetivo de controlar a qualidade dos dados coletados.

\section{Análise dos dados}

A interrupção precoce do aleitamento exclusivo ou predominante, neste estudo, identifica a situação em que a criança deixou de receber exclusiva ou predominantemente leite materno antes de completar seis meses de idade. E a duração do aleitamento materno total é compreendida como o tempo em que a criança recebe o leite materno, independente de estar consumindo qualquer outro tipo de alimento, inclusive leite de vaca.

$\mathrm{O}$ aleitamento materno predominante é o regime alimentar em que o leite materno é a principal fonte de nutrientes para criança, acompanhado somente por líquidos, a exemplo do chá e água. Enquanto que no regime de aleitamento materno complementado a criança consome o leite materno e faz uso de outros alimentos, exceto leite de vaca 9,10. Aleitamento misto caracteriza o padrão alimentar em que a criança recebe leite materno e leite de vaca ou similar, podendo ou não estar consumindo outros alimentos. E, por último, o regime de alimentação artificial, que se refere à inclusão do leite de vaca como o único alimento lácteo no regime alimentar, podendo ou não incluir outros alimentos. Adotaram-se também os indicadores propostos pela Organização Mundial da Saúde (OMS) 11 para avaliar a prevalência do aleitamento materno segundo a idade da criança no momento da entrevista, utilizandose as informações provenientes do inquérito recordatório de consumo alimentar nas 24 horas anteriores à entrevista. Com base nessas informações foram construídos também os grupos de regimes alimentares a que estavam submetidas as crianças no momento da entrevista.

O índice de condição de vida foi estratificado em categorias: elevada, intermediária, baixa e muito baixa (a categoria elevada foi tomada como referência, as demais expressam riscos crescentes). As demais variáveis estão apresentadas em forma auto-explicativa nas tabelas de resultados deste estudo.

A regressão logística foi utilizada para identificar os fatores preditores da interrupção precoce do aleitamento exclusivo ou predominante. Neste modelo de análise a variável dependente é representada, na forma dicotômica (sim/não), pela interrupção precoce do aleitamento exclusivo ou predominante. As demais variáveis são tratadas como independentes ou explanatórias.

As estimativas da duração mediana do aleitamento materno total, predominante e exclusivo, foram calculadas utilizando-se a técnica estatística de análise de sobrevivência. A associação entre as variáveis de interesse e o índice de condições de vida foi avaliada pelas estatísticas do qui-quadrado de Pearson.

Levando-se em conta o desenho amostral, baseado no conglomerado em três estágios, ado- 
tou-se a análise ponderada dos dados, que contempla a chance diferenciada do indivíduo ser selecionado para compor a amostra. Assim, utilizou-se o módulo CSAMPLE do Epi Info para a análise descritiva das variáveis, e o programa Stata para realização das estatísticas do quiquadrado de Pearson e para a análise de regressão logística.

Adotou-se a significância estatística de 0,20 para a entrada das variáveis no modelo logístico; essa probabilidade foi estipulada para que possíveis preditoras do evento não fossem excluídas da análise 12, e um valor de $\mathrm{p}<0,05$ para aceitar as associações de interesse.

\section{Resultados}

A idade da maioria das mães $(75,4 \%)$ situava-se entre 20 e 34 anos, seguidas por aquelas menores de 20 anos $(14,1 \%)$ e pelas de 35 anos ou mais (10,5\%), com média de idade de 23,8 anos $( \pm 9,8$ DP). A maioria das mães $(68,2 \%)$ cursou até o 2o ano do 2o grau e 31,8\% delas concluíram o segundo grau. Dentre as crianças estudadas, $46,9 \%$ tinham de 12 a 24 meses, 30,4\% de 6 a 12 meses e $22,7 \%$ eram menores de 6 meses. Quanto ao sexo, observou-se um pequeno predomínio do sexo feminino (51,0\%).

A caracterização da escolaridade materna, da assistência no pré e pós-natal prestada às mães e crianças e da prática alimentar de acordo com o ICV, está apresentada na Tabela 1 . Observou-se que à medida que o índice de condições de vida se elevava, aumentava também o nível de escolaridade materna $(p=0,0003)$. A busca do serviço pré-natal antes dos três meses de gestação foi referida por $65,5 \%$ das mulheres deste estudo; sendo que, a procura pelo serviço de saúde foi feita mais precocemente pelas mulheres que possuem melhores condições sociais e econômicas $(78,3 \%)$, diminuindo gradativamente conforme pioravam as condições de vida $(p=0,006)$. O atendimento ao parto ocorreu na primeira maternidade procurada para $85,9 \%$ das gestantes do estrato de elevada condição de vida e diminuiu à proporção que essas condições se tornam mais adversas, atingindo somente $67,5 \%$ daquelas cujo índice condições de vida foi classificado como muito baixo; verificou-se que $12,1 \%$ das mulheres que tinham boas condições econômicas (ICV elevado) não foram atendidas na primeira maternidade procurada, contra $32,5 \%$ daquelas que viviam em situações altamente desfavoráveis (ICV muito baixo) ( $\mathrm{p}=0,0203)$. O local de permanência da criança após o nascimento $(\mathrm{p}=0,0194) \mathrm{e}$ a orientação sobre o aleitamento materno na maternidade ( $\mathrm{p}=0,0211)$ variaram significantemente com as condições sociais e econômicas da família.

O oferecimento precoce de alimentos diferentes do leite materno antes dos seis meses de idade foi referido para $83,3 \%$ das crianças estudadas. Observou-se também que a prática da interrupção precoce do aleitamento exclusivo ou predominante aumentava à medida que as condições de vida se tornavam mais precárias. A interrupção precoce do aleitamento exclusivo ou predominante foi praticada por $71,8 \%$ das mães que apresentavam melhores condições de vida (ICV elevado), elevou-se progressivamente e atingiu $85,7 \%$ daquelas que tinham piores condições de vida (ICV muito baixo) ( $\mathrm{p}=$ 0,035) (Tabela 1).

Identificou-se também que o leite de vaca integral (pó, líquido, esterilizado ou pasteurizado) foi o primeiro leite diferente do materno oferecido à criança $(55,1 \%)$; o leite modificado $(44,9 \%)$ ocupou o segundo lugar na escolha materna. O leite modificado foi o leite de escolha de $72,1 \%$ das mães de melhores condições de vida, esse percentual diminuiu à proporção que essas condições se tornavam mais precárias e culminou com a escolha de $38,4 \%$ daquelas que viviam em condições de vida muito baixa. Movimento inverso foi observado para o leite de vaca, cuja escolha foi de $27,9 \%$ entre as mães de mais altas condições de vida e de $61,6 \%$ entre aquelas de condição de vida muito baixa $(\mathrm{p}=0,002)$ (Tabela 1 ).

As condições de vida das crianças e suas famílias associaram-se também com o acesso à orientação da prática da alimentação na infância. Nesse sentido, a escolha do leite a ser oferecido à criança teve a orientação de um profissional de saúde em $66,2 \%$ dos casos quando a mãe possuía melhor condição de vida (ICV elevado) e declinou para $25,5 \%$ quando essas condições eram muito baixas; enquanto o vizinho ou parente orientou a escolha de $33,8 \%$ das mães de condições de vida considerada alta, o fez para $74,5 \%$ das mães de piores condições de vida $(\mathrm{p}<0,001)$ (Tabela 1$)$.

Os resultados da análise de sobrevivência realizada para avaliar a duração mediana das diversas categorias do aleitamento materno estão apresentados na Figura 1 e na Tabela 2. Observou-se que $96,7 \%$ das crianças iniciaram a amamentação ao peito. A duração mediana do aleitamento materno exclusivo entre as crianças deste estudo foi de 30,6 dias, portanto, $50,0 \%$ delas foram amamentadas ao peito exclusivamente por cerca de um mês. Notou-se que, ao completar 120 dias de vida, $6,9 \%$ delas estavam em uso de leite materno exclusivo e, 
Escolaridade materna, assistência no pré e pós-natal prestada às mães e crianças e prática alimentar das crianças menores de 24 meses de idade $(n=811)$, de acordo com índice de condições de vida (ICV). Salvador, Bahia, Brasil, 1996.

\begin{tabular}{|c|c|c|c|c|c|c|}
\hline \multirow[t]{3}{*}{ Variáveis } & \multicolumn{4}{|c|}{ ICV } & & \\
\hline & \multirow[t]{2}{*}{ Elevado } & \multirow[t]{2}{*}{ Intermediário } & \multirow[t]{2}{*}{ Baixo } & \multirow[t]{2}{*}{ Muito baixo } & \multicolumn{2}{|c|}{ Total } \\
\hline & & & & & $n$ & $\%$ \\
\hline \multicolumn{7}{|l|}{ Escolaridade materna* } \\
\hline$\geq 3$ 으 ano (2o grau) & 54,8 & 43,0 & 24,4 & 22,9 & 213 & 31,8 \\
\hline Da 4a série (1ㅇ grau) ao 2o ano (2ㅇ grau) & 34,2 & 37,3 & 51,8 & 53,8 & 320 & 46,8 \\
\hline \multirow[t]{2}{*}{ < 4a série (1ㅇ grau) } & 11,0 & 19,6 & 23,9 & 23,3 & 144 & 21,4 \\
\hline & \multicolumn{4}{|c|}{$p=0,0003$} & & \\
\hline \multicolumn{7}{|l|}{ Início do pré-natal* (meses) } \\
\hline$\leq 3$ & 78,3 & 74,4 & 63,1 & 56,9 & 501 & 65,5 \\
\hline \multirow[t]{2}{*}{$>3$} & 21,7 & 25,6 & 36,9 & 43,1 & 270 & 34,5 \\
\hline & \multicolumn{4}{|c|}{$p=0,006$} & & \\
\hline \multicolumn{7}{|l|}{$\begin{array}{l}\text { Atendimento na primeira maternidade } \\
\text { procurada para o parto* }\end{array}$} \\
\hline Sim & 85,9 & 78,1 & 72,0 & 67,5 & 569 & 73,5 \\
\hline \multirow[t]{2}{*}{ Não } & 12,1 & 21,9 & 28,0 & 32,5 & 206 & 26,5 \\
\hline & \multicolumn{4}{|c|}{$p=0,0203$} & & \\
\hline \multicolumn{7}{|l|}{$\begin{array}{l}\text { Local de permanência da criança } \\
\text { após o nascimento* }\end{array}$} \\
\hline Alojamento conjunto & 35,8 & 43,5 & 38,1 & 47,4 & 314 & 42,6 \\
\hline Berçário e alojamento conjunto & 43,2 & 36,5 & 34,4 & 25,0 & 239 & 32,6 \\
\hline \multirow[t]{2}{*}{ Berçário/UTI } & 20,1 & 20,0 & 27,5 & 27,6 & 185 & 24,8 \\
\hline & \multicolumn{4}{|c|}{$p=0,0194$} & & \\
\hline \multicolumn{7}{|l|}{$\begin{array}{l}\text { Orientação sobre aleitamento } \\
\text { materno na maternidade* }\end{array}$} \\
\hline Sim & 73,2 & 80,1 & 70,1 & 65,4 & 543 & 71,7 \\
\hline \multirow[t]{2}{*}{ Não } & 26,8 & 19,9 & 29,9 & 34,6 & 222 & 28,3 \\
\hline & \multicolumn{4}{|c|}{$p=0,0211$} & & \\
\hline \multicolumn{7}{|l|}{$\begin{array}{l}\text { Interrupção precoce do aleitamento } \\
\text { exclusivo ou predominante }\end{array}$} \\
\hline Sim & 71,8 & 81,8 & 84,9 & 85,7 & 674 & 83,3 \\
\hline \multirow[t]{2}{*}{ Não } & 28,2 & 18,2 & 15,1 & 14,3 & 137 & 16,7 \\
\hline & \multicolumn{4}{|c|}{$p=0,035$} & & \\
\hline \multicolumn{7}{|l|}{ Primeiro leite oferecido à criança* } \\
\hline Leite de vaca integral & 27,9 & 50,9 & 58,3 & 61,6 & 383 & 55,1 \\
\hline \multirow[t]{2}{*}{ Leite modificado } & 72,1 & 49,1 & 41,7 & 38,4 & 315 & 44,9 \\
\hline & & & $p=0,002$ & & & \\
\hline Orientação para o uso do primeiro leite* & & & & & & \\
\hline Iniciativa própria vizinho/parente & 33,8 & 56,2 & 70,9 & 74,5 & 453 & 65,1 \\
\hline Profissional de saúde & 66,2 & 43,8 & 29,1 & 25,5 & 242 & 34,9 \\
\hline & & & $p<0,001$ & & & \\
\hline
\end{tabular}

* Houve perda de informações;

** Dados obtidos com base em informações sobre a época de oferecimento de alimentos complementares

e recordatório de 24 horas, sem utilizar a análise de sobrevivência. Aquelas crianças que ainda estavam em aleitamento materno exclusivo ou predominante no momento da entrevista foram classificadas na condição de "não". 
Figura 1

Duração do aleitamento materno das crianças menores de 24 meses $(n=811)$, segundo o tipo de aleitamento.

Salvador, Bahia, Brasil, 1996.

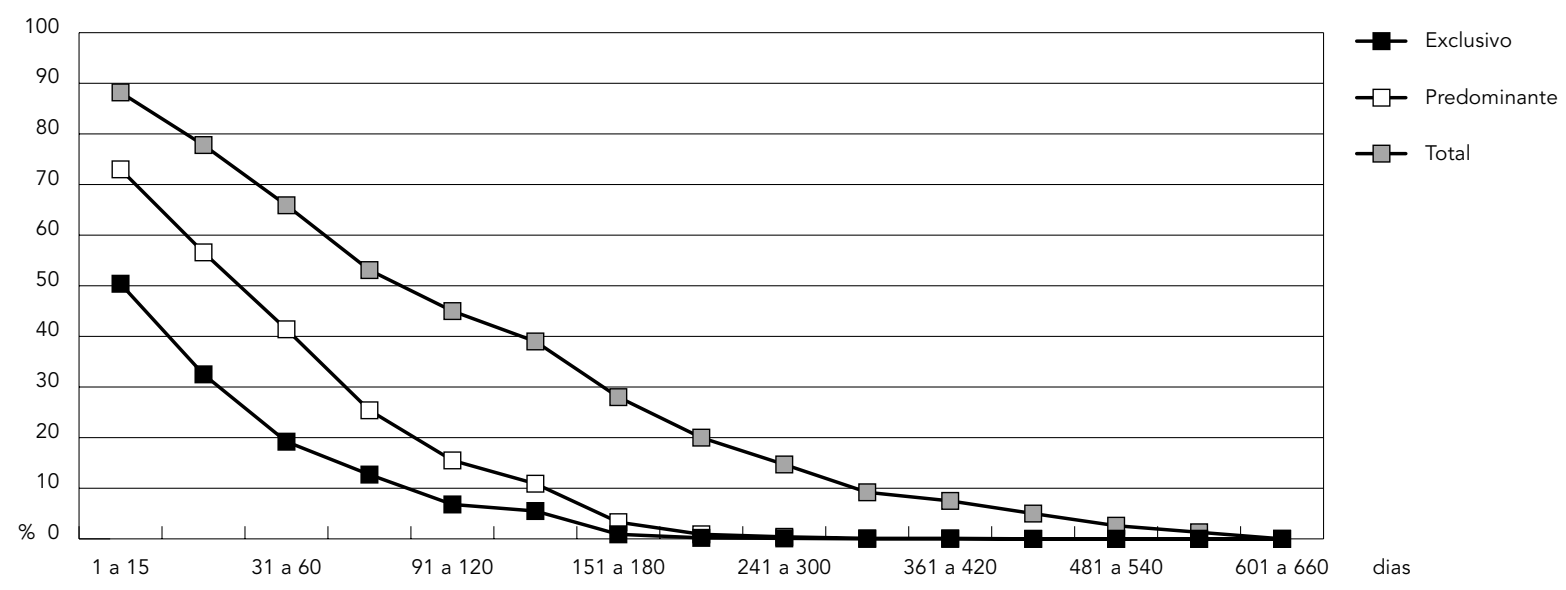

Tabela 2

Duração mediana do aleitamento materno em crianças menores de 24 meses $(n=811$ ), de acordo com índice de condições de vida (ICV). Salvador, Bahia, Brasil, 1996.

\begin{tabular}{lccc}
\hline \multirow{2}{*}{ ICV } & \multicolumn{3}{c}{ Aleitamento materno (em dias) } \\
& Exclusivo & Predominante & Total \\
\hline Elevado & 42,9 & 90,0 & 135,0 \\
Intermediário & 54,4 & 90,9 & 135,0 \\
Baixo & 26,1 & 64,5 & 124,4 \\
Muito baixo & 23,2 & 62,8 & 132,0 \\
Total & 30,6 & 73,0 & 131,5 \\
\hline
\end{tabular}

aos 180 dias, $0,9 \%$ delas praticavam esse regime alimentar. A duração mediana do aleitamento materno exclusivo diminuiu à medida que as condições de vida se tornaram mais precárias. Crianças que viviam em condições de vida consideradas elevadas foram amamentadas exclusivamente ao peito por 42,9 dias e no ICV intermediário por 54,4 dias, enquanto aquelas com condições de vida classificadas como baixa e muito baixa foram amamentadas exclusivamente ao peito por 26,1 e 23 dias, respectivamente (Tabela 2 ).

A duração mediana do aleitamento materno predominante para o conjunto das crianças investigadas foi de 73 dias. Aos 120 e 180 dias de vida esse regime alimentar era praticado, respectivamente, por $15,5 \%$ e $3,3 \%$ das crianças (Figura 1). Identificou-se que o tempo do aleitamento materno predominante para as crianças oriundas de famílias de condições de vida elevada e intermediária foi, respectivamente, 90,0 e 90,9 dias; enquanto aquelas com condições de vida baixa e muito baixa ficaram submetidas a esse regime alimentar por, respectivamente, 64,5 e 62,8 dias (Tabela 2).

A duração mediana do aleitamento total foi de 131,5 dias para o total das crianças investigadas. Assim, o leite de vaca e outros alimentos complementares já integravam o cardápio de $50,0 \%$ dessas crianças aos quatro meses de idade (Tabela 2). Verificou-se ainda que, ao completar um ano de vida, 9,2\% delas recebiam leite materno (Figura 1). A duração mediana do aleitamento materno total foi similar (135 dias), entre as crianças de famílias com condições econômicas mais vantajosas (ICV elevado e intermediário); enquanto a mediana de 124,4 dias foi observada para aquelas que tinham condições de vida baixa e de 132 dias quando a condição de vida foi classificada como muito baixa (Tabela 2).

Na Figura 2, é apresentada a caracterização do regime alimentar no momento da entrevista, com base no inquérito de consumo recordatório de 24 horas e a época de oferecimento 
Condição da alimentação das crianças menores de 24 meses ( $n=811$ ) no momento da entrevista, segundo a faixa etária. Salvador, Bahia, Brasil, 1996.
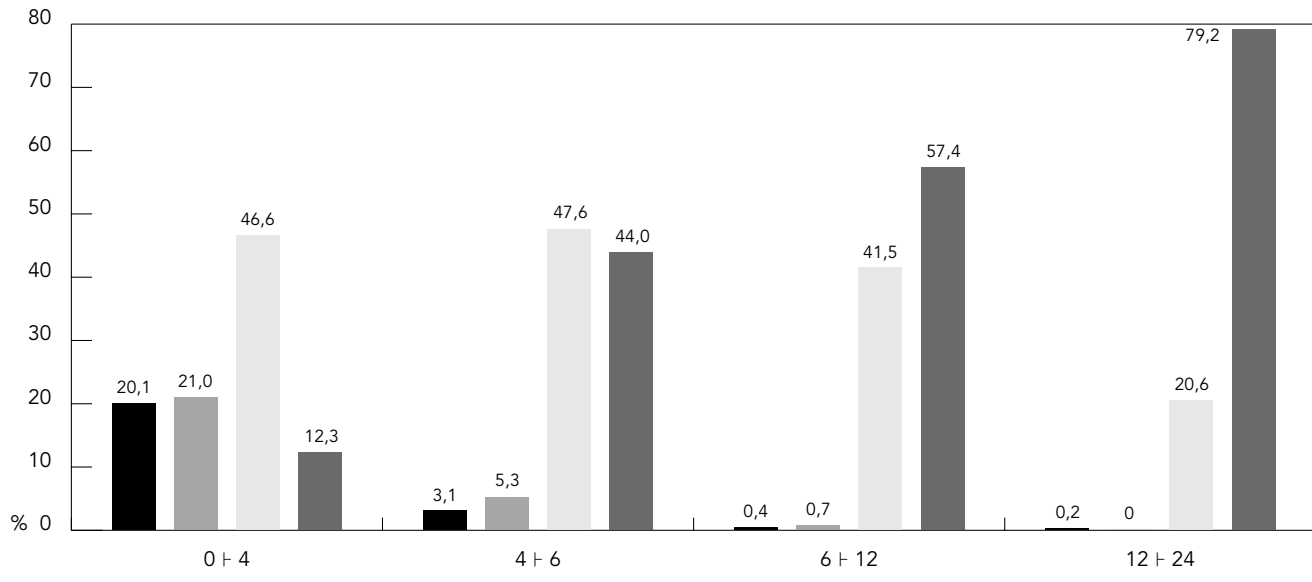

Aleitamento materno exclusivo

Aleitamento materno predominante

Aleitamento materno complementado e misto

Alimentação artificial

de alimentos complementares, independentemente das condições de vida das crianças e suas famílias. Observou-se que, antes do quarto mês de vida, $20,1 \%$ das crianças estavam sendo amamentadas exclusivamente ao peito, $21,0 \%$ delas estavam em regime de aleitamento materno predominante e $46,6 \%$ já haviam consumido outros alimentos diferentes do leite materno. As prevalências do aleitamento exclusivo e predominante diminuíram para $3,1 \%$ e $5,3 \%$, respectivamente, entre o quarto e o sexto mês de vida da criança. A partir dos seis meses, mais da metade das crianças $(57,4 \%)$ já consumiam o leite de vaca, regime de alimentação artificial, percentual que se eleva para 79,2\% entre os 12 a 24 meses de vida. Nesta mesma faixa etária o regime do aleitamento materno complementado e misto foi identificado para $20,6 \%$ delas.

$\mathrm{O}$ odds ratio (OR) para a associação entre a interrupção precoce do aleitamento exclusivo ou predominante e as variáveis sócio-econômicas, demográficas e de cuidados oferecidos às mães e às suas crianças estão apresentados na Tabela 3. A partir dos resultados da análise logística multivariada (OR ajustado), observouse que as crianças de famílias que estavam submetidas às condições de vida consideradas baixas tinham 2,3 vezes mais chances de serem desmamadas precocemente (IC95\%: 1,09-5,01), elevando-se para 2,5 quando as condições de vida eram muito baixas (IC95\%: 1,20-5,34), quan- do consideradas aquelas que tinham melhores condições de sobrevivência.

A idade materna também se mostrou associada à interrupção precoce do aleitamento exclusivo ou predominante. Os filhos de mães menores de 20 anos de idade têm 2,2 vezes mais chances de serem desmamados (IC95\%: 1,283,85 ) antes dos 6 meses de vida quando comparados àqueles de mães com idade entre $20 \mathrm{a}$ 34 anos. Esse risco permaneceu após o ajuste pelas demais variáveis (OR ajustado $=2,2$; IC95\%: 1,07-4,35). As demais variáveis incluídas no modelo não se mostraram associadas ao evento estudado.

\section{Discussão}

Os resultados deste estudo tornam evidente a forte associação entre as condições de vida e a prática alimentar nos dois primeiros anos de vida. As precárias condições de vida (ICV baixo e muito baixo) associaram-se à interrupção precoce do aleitamento exclusivo ou predominante. A criança sob condições de vida consideradas baixas tem 2,3 vezes mais chances de realizar a introdução precoce de alimentos complementares no esquema alimentar; essa chance aumenta para 2,5 quando as condições de vida são muito baixas. Tais achados concordam com outros estudos que relacionam baixas condições sócio-econômicas da 
Odds ratio (OR) para a associação entre a interrupção precoce do aleitamento exclusivo ou predominante e as variáveis sócio-econômicas, demográficas e de cuidados oferecidos às mães e às crianças menores de 24 meses de idade ( $n=811)$. Salvador, Bahia, Brasil, 1996.

\begin{tabular}{|c|c|c|c|c|}
\hline Variáveis & OR bruta & IC95\% & OR ajustada* & IC95\% \\
\hline \multicolumn{5}{|l|}{ Índice de condição de vida (ICV) } \\
\hline Elevado & 1,00 & & 1,00 & \\
\hline Intermediário & 1,77 & $0,94-3,32$ & 1,89 & $0,88-4,03$ \\
\hline Baixo & 2,22 & $1,19-4,13$ & 2,34 & $1,09-5,01$ \\
\hline Muito baixo & 2,35 & $1,25-4,42$ & 2,52 & $1,20-5,34$ \\
\hline \multicolumn{5}{|l|}{ Idade materna (anos) } \\
\hline $20-34$ & 1,00 & & 1,00 & \\
\hline$<20$ & 2,22 & $1,28-3,85$ & 2,16 & $1,07-4,35$ \\
\hline$\geq 35$ & 1,49 & $0,78-2,85$ & 1,27 & $0,59-2,74$ \\
\hline \multicolumn{5}{|l|}{ Escolaridade materna** } \\
\hline$\geq 3$ 으 ano (2o grau) & 1,00 & & 1,00 & \\
\hline Da 4a série (1ㅇ grau) ao 2o ano (2o grau) & 1,42 & $0,90-2,21$ & 0,99 & $0,59-1,65$ \\
\hline$<4$ a série (1ㅇgrau) & 1,43 & $0,75-2,72$ & 1,05 & $0,48-2,28$ \\
\hline \multicolumn{5}{|l|}{ Sexo do recém-nascido } \\
\hline Feminino & 1,00 & & 1,00 & \\
\hline Masculino & 1,23 & $0,82-1,84$ & 1,51 & $0,94-2,42$ \\
\hline \multicolumn{5}{|l|}{ Peso ao nascer $(\mathbf{g})^{\star \star}$} \\
\hline$\geq 2.500$ & 1,00 & & 1,00 & \\
\hline$<2.500$ & 1,43 & $0,89-2,31$ & 0,94 & $0,50-1,74$ \\
\hline \multicolumn{5}{|l|}{ Amamentação na sala de parto } \\
\hline Sim & 1,00 & & 1,00 & \\
\hline Não & 1,35 & $0,70-2,58$ & 1,47 & $0,74-2,93$ \\
\hline \multicolumn{5}{|l|}{ Início do pré-natal (meses) } \\
\hline$\leq 3$ & 1,00 & & 1,00 & \\
\hline$>3$ & 1,43 & $0,91-2,23$ & 1,33 & $0,77-2,85$ \\
\hline \multicolumn{5}{|l|}{$\begin{array}{l}\text { Atendimento na primeira maternidade } \\
\text { procurada para o parto }\end{array}$} \\
\hline $\operatorname{Sim}$ & 1,00 & & 1,00 & \\
\hline Não & 0,89 & $0,58-1,37$ & 0,74 & $0,44-1,26$ \\
\hline \multicolumn{5}{|l|}{ Local onde a criança ficou após o nascimento } \\
\hline No quarto junto com a mãe & 1,00 & & 1,00 & \\
\hline Berçário e no quarto com a mãe & 0,89 & $0,57-1,39$ & 1,15 & $0,69-1,91$ \\
\hline Berçário/UTI & 1,45 & $0,91-2,30$ & 1,80 & $0,96-3,38$ \\
\hline \multicolumn{5}{|c|}{ Orientação sobre aleitamento na maternidade } \\
\hline Sim & 1,00 & & 1,00 & \\
\hline Não & 1,27 & $0,83-1,96$ & 1,35 & $0,77-2,34$ \\
\hline
\end{tabular}

* Ajustada para demais variáveis incluídas no modelo;

** Não alcançaram significância estatística no stepwise $(0,20)$, mas foram consideradas importantes para ajuste do modelo final. 
família com menor duração do aleitamento materno 13,14 .

No entanto, independentemente da condição sócio-econômica das famílias das crianças de Salvador, a duração do aleitamento materno, em quaisquer de suas modalidades, foi extremamente baixa, na medida em que as medianas para o aleitamento exclusivo e predominante foram, respectivamente, de 30,6 e de 73 dias, e para o aleitamento total de 131,5 dias, valores mais baixos do que o observado para todo o Brasil 15 (aproximadamente 210 dias) na mesma época em que foi realizado este estudo. Embora a taxa de iniciação do aleitamento materno tenha sido semelhante $(96,7 \%)$ à observada para o Brasil como um todo $(97,0 \%)$, nessa mesma época 15.

$\mathrm{Na}$ atualidade tem sido destacada tendência de aumento progressivo na duração do aleitamento materno total para o Brasil em geral. Embora os estudos que indiquem essa tendência envolvam crianças de diferentes faixas etárias, os resultados para o aleitamento materno total são potencialmente comparáveis. Essa tendência de aumento na duração do aleitamento materno total foi também observada em Salvador. A duração mediana para o aleitamento total de 106 dias registrada pela Secretaria de Saúde do Estado da Bahia em 1992 16, elevou-se ligeiramente para 131,5 dias em 1996 quando foi realizado este estudo, e em 1999 é observado aumento expressivo atingindo 274,6 dias de duração, conforme mediana calculada pelo estudo da prevalência do aleitamento materno nas capitais e Distrito Federal 17.

No entanto, resultados curiosos são observados para a prevalência e duração do aleitamento materno exclusivo na cidade de Salvador. Nota-se que no período de 1996/1999 a taxa do aleitamento materno exclusivo aumentou de $20,1 \%$, prevalência estimada por este estudo em 1996, para $27,0 \%$, conforme a taxa calculada pela investigação realizada nas capitais e Distrito Federal em 1999 17. Mas, curiosamente a duração mediana do aleitamento materno cai nesse período, passando de 30,6 dias em 1996, conforme se observou neste estudo, para 15,4 dias em 199917.

A tendência de aumento progressivo da prática da amamentação no Brasil vem sendo registrada desde as décadas de 70 e 80 18. Estudos mais recentes também mostram aumento no tempo médio de aleitamento materno para o Brasil como um todo. Em 1989, a Pesquisa Nacional sobre Saúde e Nutrição (PNSN) 19 identificou duração mediana do aleitamento materno total de 134 dias, e em 1996, na Pesquisa Nacional sobre Demografia e Saúde (PNDS), um registro de aproximadamente 210 dias 15. Contudo, essa tendência de aumento não foi registrada para o aleitamento materno exclusivo, cujo declínio de 72 para 31 dias foi registrado, respectivamente, pela PNSN (1989) e PNDS (1996). Vários investigadores têm observado ainda que a prevalência do aleitamento materno exclusivo raramente alcançou índices superiores a $30,0 \%$ em diferentes grupos populacionais brasileiros 20,21,22,23,24.

Ignorando eventuais diferenças metodológicas adotadas nos estudos, a comparação dos resultados desta investigação com aqueles da prevalência do aleitamento materno nas capitais e no Distrito Federal, sugere aumento expressivo na prevalência e duração do aleitamento materno total em Salvador 17, sem, contudo, atingir a metade da duração mediana de 728 dias preconizada pela OMS e pelo Ministério da Saúde do Brasil 10,17. Embora tenha sido registrado também um aumento discreto da prevalência do aleitamento materno exclusivo nesta capital, identificou-se declínio na duração mediana no período de 1996 a 1999.

Salienta-se, no entanto, que esses estudos não avaliaram a distribuição da duração e da prevalência do aleitamento materno, nas suas diferentes modalidades, segundo os estratos sociais em que estão inseridas as crianças e suas famílias. Neste sentido, os resultados deste estudo indicam que as más condições de vida familiar constituem fatores de risco para interrupção precoce do aleitamento exclusivo ou predominante, com o conseqüente oferecimento de alimentos diferentes do leite materno à criança. Esses resultados são observados tanto na análise bivariada, como no modelo multivariado que contempla o efeito conjunto das variáveis preditoras do modelo.

Os resultados deste estudo indicam ainda que as ações de promoção do aleitamento materno devem atingir as crianças indistintamente da condição social, mas enfoque especial e possivelmente diferenciado deve ser adotado, dado que os fatores que se associam ao abandono precoce do aleitamento materno se diferenciam de acordo com as condições materiais de vida da criança e de sua família.

Entende-se que o leite materno seja altamente benéfico para qualquer criança, independentemente da classe social a qual pertença, dado a proteção contra doenças conferida por esse leite e a adequação à fisiologia digestiva, sistema renal, neurológico e imunológico 10; e para a criança pobre esse efeito protetor reveste-se de importância singular, na medida em que essas crianças estão expostas aos efeitos adversos da iniqüidade social e econômica, e às mais elevadas cargas de morbidade. 
O oferecimento precoce de alimentos complementares está associado com a maior ocorrência de anemia 25 , doenças infecciosas, particularmente gastrintestinais e respiratórias 10 , e comprometimento no crescimento físico da criança 26 . Nesse sentido, as crianças pobres são as que mais se beneficiam das vantagens nutricionais e imunológicas conferidas pelo leite materno, na medida em que a restrita disponibilidade de recursos financeiros intrafamiliar impede prover o acesso ao alimento e à saúde, e limita a qualidade e a quantidade de cuidados dispensados à criança no seio da família 27,28.

Na condição imperiosa de impedimento para a prática do aleitamento materno no primeiro ano de vida, o leite de escolha deve recair sobre o modificado 29. Mas, para as crianças que integram este estudo, o leite de escolha é o de vaca integral, cujo uso aumenta à proporção que pioram as condições de vida da criança e de sua família. Esse resultado indica que o tipo de leite oferecido à criança guarda uma estreita relação com a condição social e econômica da família. Assim, à medida que pioram as condições de vida, cai a escolha do leite modificado, como o primeiro leite diferente do leite de peito a ser oferecido à criança ( $p=0,002)$, cujo preço de mercado é mais elevado, e declina também a orientação prestada pelo profissional de saúde sobre a indicação do tipo do leite recomendado à criança $(\mathrm{p}<0,001)$. Nesse cenário, a desigualdade em saúde se manifesta também de forma contundente no acesso diferenciado ao alimento, na disponibilidade de orientação nutricional e no padrão alimentar dessa população.

Em que pesem as intervenções sugeridas pelos organismos internacionais a exemplo da OMS, Fundo das Nações Unidas para a Infância (UNICEF) e Banco Mundial 1,2,3, para minimizar o impacto das adversidades sociais e econômicas sobre as condições de saúde das populações pobres, persistem ainda as diferenças marcantes no acesso e na qualidade dos serviços prestados entre os diferentes estratos sociais. No contexto das desigualdades sociais e no restrito acesso à orientação alimentar e nutricional por profissional qualificado, as crianças estão submetidas a regimes alimentares marcados pelo oferecimento precoce de alimentos complementares. Assim, antes dos quatro meses de idade $46,6 \%$ delas já se encontravam em regime de aleitamento materno complementado e misto e $12,3 \%$ já tinham o seu regime alimentar integrado somente pelo leite de vaca. Entre os 6 e 12 meses, 57,4\% já consumiam leite de vaca, percentual que se eleva para $79,2 \%$ entre 12 e 24 meses de idade. Nesse sentido, os riscos à saúde pela adoção dessa prática alimentar podem ser eminentes. Esse padrão de consumo é similar ao registrado por Silveira \& Lamounier 30 e Assis et al. 31 , para outros grupos de crianças brasileiras.

Os resultados deste estudo realçam também a relação entre os cuidados prestados às gestantes, crianças e puérperas e as condições materiais de vida. Assim, à medida que pioram as condições de vida declina o percentual de busca precoce ao serviço pré-natal $(\mathrm{p}=0,006)$ e diminui o percentual de atendimento na primeira maternidade procurada para o parto $(\mathrm{p}=$ 0,0203), indicando a insuficiente oferta ou a baixa qualidade do serviço, ou o desconhecimento materno da importância dessas condições para a saúde materno-infantil.

Nesse sentido, as crianças pobres estão expostas a fatores desvantajosos que as impedem de ter acesso a esses serviços e de receber as intervenções efetivas que garantem adequadas condições de saúde e nutrição, quando comparadas com aquelas de melhores estratos sócio-econômicos. As evidências mostram também que a promoção das ações não atinge a todas as crianças que delas necessitam 4 , fazendo sobressair a limitada distribuição e acesso aos serviços, e mostram também que em muitas situações a qualidade do serviço prestado limita a eficiência das ações ofertadas e impede também o cumprimento de ações básicas de promoção à saúde e nutrição.

Curiosamente, com exceção da idade materna, outras variáveis incluídas no modelo multivariado não foram identificadas como predisponentes à interrupção precoce do aleitamento exclusivo ou predominante. Neste leque de variáveis, estão incluídas principalmente aquelas inerentes ao comportamento materno ante a gestação e aquelas que dizem respeito ao serviço prestado pelo sistema de saúde. Possivelmente, quando uma variável que exprime as condições de vida é considerada no conjunto de potenciais preditores da prática do aleitamento materno, esta passa a exercer um efeito mais expressivo sobre o evento do que as demais consideradas isoladamente.

No entanto, vale comentar que poucos são os estudos que exploram as condições econômicas $18,32,33,34$, conjuntamente com as variáveis da esfera biológica e com aquelas dos serviços de saúde, enquanto preditoras da prática do aleitamento materno e da alimentação na infância. Além disso, muitos dos estudos adotam como amostras grupos selecionados, condições que podem distorcer os resultados.

À guisa de conclusão pode-se comentar que, quando as precárias condições restringem a 
possibilidade e a oportunidade da escolha materna sobre a adoção da prática do aleitamento materno e dos regimes alimentares a serem adotados para suas crianças, induzem precocemente aos riscos da alimentação qualitativa e quantitativamente inadequadas.

Assim, os resultados deste estudo remetem à reflexão de que as políticas direcionadas à promoção da prática adequada do aleitamento materno e da adoção da alimentação saudável na infância devem considerar a relevância dos fatores sociais e econômicos relacionados ao evento, e apontam também a necessidade de contemplar políticas voltadas para a redução das desigualdades no campo da atenção à saúde.

\section{Resumo}

Este trabalho tem como objetivo conhecer a duração do aleitamento materno, regime alimentar e fatores associados segundo condições de vida em Salvador, Bahia, Brasil. Este estudo de prevalência envolve 811 crianças menores de 24 meses. Para analisar os dados utilizou-se análise de sobrevivência, qui-quadrado de Pearson e regressão logística. A duração mediana do aleitamento exclusivo, predominante e total foi de 30,6, 73 e 131,5 dias, respectivamente. Identificou-se que 83,3\% das crianças tiveram interrupção precoce do aleitamento exclusivo ou predominante. As crianças de famílias com condições de vida consideradas baixas tinham 2,3 vezes mais chances (IC95\%: 1,095,01) de consumirem alimentos complementares precocemente; essa chance se elevou para 2,5 (IC95\%: 1,20-5,34) entre aquelas de famílias com condições de vida muito baixas. A interrupção precoce do aleitamento exclusivo ou predominante associou-se com a maternidade em idade precoce e as precárias condições de vida das crianças e suas famílias. Programas direcionados à promoção da prática adequada do aleitamento materno e adoção da alimentação saudável na infância devem considerar a relevância dos fatores sociais envolvidos no oferecimento precoce de alimentos complementares à criança.

Bem-estar da Criança; Aleitamento Materno; Hábitos Alimentares

\section{Colaboradores}

G. S. S. Gomes participou da elaboração e implementação da análise estatística. A. M. O. Assis e M. S. Prado coordenaram o trabalho de campo e, em conjunto com L. P. M. Oliveira realizaram coleta de dados, implementação e interpretação dos resultados. L. P. M. Oliveira, A. M. O. Assis e M. L. Barreto participaram da elaboração final do artigo.

\section{Referências}

1. Gwatkin DR. Health inequalities and the health of the poor: what do we know? What can we do? Bull World Health Organ 2000; 78:3-18.

2. Wagstaff A. Socioeconomic inequalities in child mortality: comparisons across nine developing countries. Bull World Health Organ 2000; 78:19-29.

3. Navarro V. The world situation and WHO. Lancet 2004; 363:1321-3.

4. Jones G, Steketee RW, Black RE, Bhutta ZA, Morris SS, Bellagio Child Survival Study Group. How many child deaths can we prevent this year? Lancet 2003; 362:65-71.

5. World Health Organization. Physical status: the use and interpretation of antropometry. Report of a WHO Expert Committee. Geneva: World Health Organization; 1995.

6. Javorski M, Scochi CGS, Lima RAG. Os programas nacionais de incentivo ao aleitamento materno: uma análise crítica. Pediatr Mod 1999; 35:30-6.

7. Claeson M, Gillespie D, Mshinda H, Troedsson H, Victoria CG, Bellagio Study Group on Child Survival. Knowledge into action for child survival. Lancet 2003; 362:323-7.

8. Assis AMO, Barreto ML, Santos LMP, Sampaio LR, Magalhães LP, Prado MS, et al. Condições de vida, saúde e nutrição na infância na Cidade de Salvador. 1a Ed. Salvador: Bureau, Gráfica e Editora; 2000.

9. Organización Panamericana de la Salud, Organización Mundial de la Salud. Indicadores para evaluar las practicas de lactancia materna. Washington DC: Organización Panamericana de la Salud, Organización Mundial de la Salud; 1991.

10. World Health Organization. Complementary feeding of young children in developing countries: a review of current scientific knowledge. Geneva: World Health Organization; 1998.

11. World Health Organization. Division of diarrhoeal and acute respiratory disease control: indicators for assessing breast-feeding practices. Geneva: World Health Organization; 1991. 
12. Hosmer DW, Lemenshow S. Applied logistic regression. New York: Johns Wiley \& Sons; 1989.

13. Victora CG, Huttly SRA, Barros FC, Lombardi C, Vaughan JP. Maternal education in relation to early and late child health outcomes: findings from a Braziliam cohort study. Soc Sci Med 1992; 34:899-905.

14. Rea MF, Venâncio SI, Batista LE, Santos RG, Greiner T. Possibilidades e limitações da amamentação entre mulheres trabalhadoras formais. Rev Saúde Pública 1997; 31:149-56.

15. Sociedade Civil Bem-estar Familiar no Brasil. Pesquisa nacional sobre demografia e saúde 1996. 2a Ed. Rio de Janeiro: Sociedade Civil Bem-estar Familiar no Brasil; 1999.

16. Secretaria da Saúde do Estado da Bahia. Diagnóstico de saúde das crianças e mulheres da Bahia. Rev Baiana Saúde Pública 1993; 20 Suppl 1:12-55.

17. Ministério da Saúde. Pesquisa de prevalência do aleitamento materno nas capitais e no Distrito Federal. Brasília: Ministério da Saúde; 2001.

18. Venancio SI, Monteiro CA. A tendência da pratica da amamentação no Brasil nas décadas de 70 e 80. Rev Bras Epidemiol 1998; 1:40-9.

19. Leão MM, Coitinho DC, Recine E, Costa LAL, Lacerda AJ. O perfil do aleitamento materno no Brasil. In: Instituto Brasileiro de Geografia e Estatística/Fundo das Nações Unidas para a Infância/Instituto Nacional de Alimentação e Nutrição, organizadores. Perfil estatístico de crianças e mães no Brasil: aspectos de saúde e nutrição de crianças no Brasil, 1989. Rio de Janeiro: Instituto Brasileiro de Geografia e Estatística; 1992. p. 97110.

20. Nejar FF, Segall-Corrêa AM, Réa MF, Vianna RPT, Panigassi G. Padrões de aleitamento materno e adequação energética. Cad Saúde Pública 2004; 20:64-71.

21. Vieira GO, Silva LR, Vieira TO, Almeida JAG, Cabral VA. Hábitos alimentares de crianças menores de 1 ano amamentadas e não-amamentadas. J Pediatr 2004; 80:411-6.

22. Figueiredo MG, Sartorelli DS, Zan TAB, Garcia E, Silva LC, Carvalho FLP, et al. Inquérito de avaliação rápida das práticas de alimentação infantil em São José do Rio Preto, São Paulo, Brasil. Cad Saúde Pública 2004; 20:172-9.

23. Venancio SI, Escuder MM, Kitoko P, Réa MF, Monteiro CA. Freqüência e determinantes do aleitamento materno em municípios do Estado de São Paulo. Rev Saúde Pública 2002; 36:313-8.

24. Kitoko PM, Réa MF, Venancio SI, Vasconcelos ACCP, Santos EKA, Monteiro CA. Situação do aleitamento materno em duas capitais brasileiras: uma análise comparada. Cad Saúde Pública 2000; 16:1111-9.
25. Assis AMO, Gaudenzi EN, Gomes G, Ribeiro RC, Szarfarc SC, Souza SB. Níveis de hemoglobina, aleitamento materno e regime alimentar no primeiro ano de vida. Rev Saúde Pública 2004; 38: 543-51.

26. Piwoz EG, Kanashiro HC, Romaña GL, Black RE, Brown KH. Feeding practices and growth among low income Peruvian infants: a comparison of internationally-recommended definitions. Int J Epidemiol 1996; 25: 103-14.

27. Islam R. Poverty and its effect on nutrition: some questions based on the Asian experience. In: Administrative Committee on Coordination, Sub Committee on Nutrition. Nutrition and poverty. Geneva: World Health Organization; 1997. p. 6976. (Nutrition policy paper 16).

28. Chirmulay D. Child, nutrition and poverty. In: Administrative Committee on Coordination, Sub Committee on Nutrition. Nutrition and poverty. Geneva: World Health Organization; 1997. p. 7783. (Nutrition policy paper 16).

29. Carver JD. Advances in nutritional modifications of infant formulas. Am J Clin Nutr 2003; 77:1550S$4 \mathrm{~S}$.

30. Silveira FJF, Lamounier JA. Prevalência do aleitamento materno e práticas de alimentação complementar em crianças com até 24 meses de idade na região do Alto Jequitinhonha, Minas Gerais. Rev Nutr 2004; 17:437-47.

31. Assis AMO, Prado MS, Freitas MCS, Silva RCR, Ramos LB, Machado AD. Prática do aleitamento materno em comunidades rurais do semi-árido baiano. Rev Saúde Pública 1994; 28:340-4.

32. Bueno BM, Souza JMP, Souza SB, Paz SMRS, Gimeno SGA, Siqueira AAF. Risco associado ao processo de desmame entre crianças nascidas em hospital universitário de São Paulo, entre 1998 e 1999: estudo de coorte prospectivo do primeiro ano de vida. Cad Saúde Pública 2003; 19:1453-60.

33. Monteiro CA, Zuniga HPP, Benício MHD, Réa MF. Estudo das condições de saúde das crianças do município de São Paulo, SP (Brasil), 1984-1985. III - Alimento materno. Rev Saúde Pública 1987; 21:13-22.

34. Horta BL, Olinto MTA, Victora CG, Barros FC, Guimarães PRV. Amamentação e padrões alimentares em crianças de duas coortes de base populacional no Sul do Brasil: tendência e diferenciais. Cad Saúde Pública 1996; 12 Suppl 1:43-8.

Recebido em 28/Out/2004

Versão final reapresentada em 16/Mar/2005

Aprovado em 03/Mai/2005 